\title{
Pregnancy, childbirth and neonatal outcomes in women with inherited bleeding disorders: a retrospective analysis.
}

\author{
Lisa Clarke ${ }^{1}$, Jennifer Curnow ${ }^{2}$, Briony Cutts $^{3}$, Bryony Ross ${ }^{4}$, and Giselle Kidson-Gerber ${ }^{5}$ \\ ${ }^{1}$ Sydney Adventist Hospital Ltd \\ ${ }^{2}$ Westmead Hospital \\ ${ }^{3}$ Royal Women's Hospital Department of Obstetrics and Gynaecology \\ ${ }^{4}$ Calvary Mater Newcastle \\ ${ }^{5}$ Prince of Wales Hospital and Community Health Services
}

June 21, 2021

\begin{abstract}
Objective To describe the characteristics and outcomes of women with inherited bleeding disorder during pregnancy and birth. Design Retrospective cohort study. Setting Tertiary care hospitals, NSW and Victoria Australia. Population 100 women with inherited bleeding disorders, who birthed 134 live infants from 132 pregnancies. Methods Data was retrospectively obtained from the patient and neonatal medical records. Descriptive analysis was used to report maternal and pregnancy characteristics, birth and neonatal outcomes. Main outcome measures Factor replacement, neuraxial analgesia use and complications, postpartum haemorrhage and neonatal complications. Results PPH occurred in $22 \%$ of deliveries with primary PPH occurring in $20 \%$ and secondary PPH in $4 \%$ of births. $48 \%$ of PPHs were classified as major. PPHs occurred across the spectrum of IBD and was evenly distributed between women who had "normalised" their factor levels in pregnancy compared to those requiring factor at the time of birth. An obstetric cause was identified in more than half of PPHs. Conclusions Women with inherited bleeding disorders can deliver safely and receive neuraxial analgesia without complication when best practices are adhered to. PPH appears to occur at higher rates than the general population despite adequate factor levels or planned replacement. Whilst an obstetric cause was demonstrable in the many cases, these findings raise concern over the current definition of "adequate" factor levels at the time of birth.
\end{abstract}

Pregnancy, childbirth and neonatal outcomes in women with inherited bleeding disorders: a retrospective analysis.

Dr Lisa Clarke ${ }^{1,2}$, Dr Jennifer Curnow, ${ }^{3,4}$ Dr Briony Cutts $^{5,6}$, Dr Bryony Ross ${ }^{7,8}$ and Dr Giselle KidsonGerber $^{9,10}$.

1. Department of Haematology, Sydney Adventist Hospital, Sydney, Australia

2. Transfusion Policy and Education, Australian Red Cross Lifeblood, Sydney, Australia

3. Department of Haematology, Westmead Hospital, Sydney, Australia

4. Faculty of Medicine and Health, University of Sydney, Sydney, Australia

5. Department of Obstetrics, Royal Women's Hospital, Melbourne, Australia

6. Department of Obstetric Medicine, Joan Kirner Women's and Children's at Sunshine Hospital, Melbourne, Australia.

7. Department of Haematology, Calvary Mater Newcastle, Australia

8. NSW Health Pathology, North (Hunter), Newcastle, Australia

9. Department of Haematology, Prince of Wales Hospital, Sydney, Australia

10. Royal Hospital for Woman, Sydney, Australia 
Corresponding author: Dr Lisa Clarke

Australia Red Cross Lifeblood

Sydney Processing Centre

17 O'Riordan street Alexandria 2015

LClarke@redcrossblood.org.au

$+61411790679$

Running title: Obstetric outcomes in inherited bleeding disorders

Abstract

Objective

To describe the characteristics and outcomes of women with inherited bleeding disorder during pregnancy and birth.

Design

Retrospective cohort study.

Setting

Tertiary care hospitals, NSW and Victoria Australia.

Population

100 women with inherited bleeding disorders, who birthed 134 live infants from 132 pregnancies.

Methods

Data was retrospectively obtained from the patient and neonatal medical records. Descriptive analysis was used to report maternal and pregnancy characteristics, birth and neonatal outcomes.

Main outcome measures

Factor replacement, neuraxial analgesia use and complications, post-partum haemorrhage and neonatal complications.

Results

PPH occurred in $22 \%$ of deliveries with primary PPH occurring in $20 \%$ and secondary PPH in $4 \%$ of births. $48 \%$ of PPHs were classified as major. PPHs occurred across the spectrum of IBD and was evenly distributed between women who had "normalised" their factor levels in pregnancy compared to those requiring factor at the time of birth. An obstetric cause was identified in more than half of PPHs.

Conclusions

Women with inherited bleeding disorders can deliver safely and receive neuraxial analgesia without complication when best practices are adhered to. PPH appears to occur at higher rates than the general population despite adequate factor levels or planned replacement. Whilst an obstetric cause was demonstrable in the many cases, these findings raise concern over the current definition of "adequate" factor levels at the time of birth.

Keywords

Inherited bleeding disorders, pregnancy, post-partum haemorrhage

Tweetable abstract: 
Women with inherited bleeding disorders can deliver without complication when best practices are maintained.

Disclosures of interest.

Nil.

Contribution to authorship.

LJC collected data, analysed the data and wrote the manuscript.

JC critically appraised the manuscript.

$\mathrm{BC}$ collected data and critically appraised the manuscript.

BR collected data and critically appraised the manuscript

GKG conceived the idea, collected data and critically appraised the manuscript.

Ethics

Ethics approval was obtained through the human research ethics committee (SESLHD HREC 15/294).

Introduction

Pregnancy and childbirth pose unique haemostatic challenges. The associated risks and severity of potential bleeding complications are increased in women with inherited bleeding disorders (IBD) however appropriate measures can limit adverse outcomes. Guidelines are available to direct management of such women but there remains a paucity of published outcome data.

IBD affect approximately 1\% of the population(1). In Australia 2643 women are registered with the Australian Bleeding Disorders Registry, with approximately $2 / 3$ of reproductive age.(2)

Haemophilia A (HA) and B (HB) are X linked disorders, resulting in deficiencies of factors VIII (FVIII) and IX (FIX) respectively.(1) Most women are asymptomatic (carriers), however women with levels $<40 \mathrm{U} / \mathrm{dL}$ are classified as having mild disease.(3) In contrast to FIX levels, FVIII levels increase significantly throughout pregnancy and exceed recommended thresholds for birth in the majority of women. $(3,4)$ Maintaining factor levels $>50 \mathrm{U} / \mathrm{dL}$ are currently recommended for birth and 3-7 days post-partum depending on mode of birth(4). Neuraxial analgesia is deemed safe when levels are above this threshold.(4) Recombinant FVIII or FIX concentrates are the treatments of choice when factor levels are inadequate in HA and HB.(5)

von Willebrand disease (VWD) is the most common IBD and can be divided into 3 types(5). Types 1 and 3 represent quantitative defects of increasing magnitude whereas Type 2 represents a qualitative defect and can be further classified into Types 2A, B, M and N depending on the mechanism of disordered haemostasis. VWD has autosomal dominant inheritance in the less severe forms and autosomal recessive inheritance in the more severe types.(5) von Willebrand Factor (VWF) levels also rise in pregnancy. This increase is generally adequate in women with Type 1, fails to overcome the functional impairment in Type 2 and is insufficient in Type 3.(4) VWF levels (or activity) $>50 \mathrm{U} / \mathrm{dL}$ are recommended for birth, neuraxial analgesia, and 3-7 days post-partum.(4) Desmopressin, which releases endogenous VWF, or VWF containing concentrates can be administered to increase VWF levels.(5)

Rare bleeding disorders include disorders of prothrombin, fibrinogen, Factor V, VII, X, XI, XIII and functional platelet disorders. There is limited data to guide management of these woman. In many, pregnancy does not improve the bleeding risk ${ }^{(3)}$ and factor replacement is achieved with transfusion of plasma, factor concentrates or platelets.(5)

Objective

We aim to describe the characteristics and outcomes of pregnancies and births of a cohort of Australian women with IBD. 


\section{Methods}

All women with a known history of an IBD, who received care throughout their pregnancy or birth at either Prince of Wales Hospital Sydney, The Royal Hospital for Women Sydney, Westmead Hospital Sydney, John Hunter Hospital Newcastle, Calvary Mater Newcastle, Royal Women's Hospital Melbourne and Joan Kirner Women's and Children's at Sunshine Hospital Melbourne between 2011 and 2020 were included. Ethics approval was obtained through the human research ethics committee (SESLHD HREC 15/294).

Data was retrospectively obtained from the patient and neonatal medical records. Descriptive analysis was used to report maternal characteristics, factor levels, factor replacement, pregnancy, birth and neonatal outcomes for each pregnancy. Data analysis was performed using SAS studio.

Funding was not received for this study.

Results:

The study captured 100 women with 132 pregnancies and 134 live births

Figure 1 demonstrates the normalisation of mean VWF levels in women with Type 1 VWD (VWF antigen level) compared to women with Type 2B and 2N VWD (VWF activity level); and mean factor levels in women with mild HA compared to mild HB, FXI deficiency and hypofibrinogenaemia. The mean factor levels for both HA and HB carriers were above recommended levels throughout all stages of pregnancy.

\section{Birth and post-partum}

At the time of birth the median maternal age was 32 years, haemoglobin $125 \mathrm{~g} / \mathrm{L}$ and platelet count $218 \times 10^{\wedge} 9 /$ L. Neuraxial analgesia was administered in $52(40 \%)$ births, advised against in $9(7 \%)$ and refused in $4(3 \%)$ despite documented adequate haemostatic potential. Normal vaginal birth occurred in $62 \%$ (81) of births with intervention required in $12 \%$ including forceps (9\%), scalp electrodes (1\%) and vacuum extraction (1\%). Intervention was required in 2 births of women with $\mathrm{X}$ linked conditions where the sex of the fetus was unknown and in 1 woman with a more severe form of VWD; only 1 infant was subsequently found to be affected and there were no documented complications. Caesarean sections were performed in $38 \%$ (49) of births with the decision directed by maternal choice or obstetric indications. Of these $14 \%$ were emergency caesarean sections (Table 1).

Factor replacement was administered in 4 pregnancies as prophylaxis at the time of obstetric intervention or treatment of pregnancy-related bleeding complications. Replacement factor or platelets were required at the time of 37 births. There were 2 women who received factor despite normalisation of levels in pregnancy, this included a women with Type 1 VWD and Mild HA. In both cases this was to facilitate out of hours neuraxial analgesia when urgent repeat factors levels were not available. Post-partum factor replacement was not continued for these women. In addition 3 women with hypofibrinogenaemia only received replacement at the time of birth otherwise all other women who received factor at the time of birth had it continued in the post-partum period. Two women with Type 2B VWD did not receive factor at the time of birth despite inadequate third trimester levels however factor was commenced immediately post-partum, one of these births was complicated by a PPH. There were two further women who only received factor replacement post-partum for bleeding complications despite adequate factor levels. This included a woman with Type 1 VWD who developed a wound haematoma at the caesarean section site in a previous pregnancy and a woman with Type 1 VWD who developed a perineal haematoma. Only 1 woman who received platelets at the time of birth for an inherited platelet disorder required ongoing platelet support post-partum.

Only $36 \%$ of women received antifibrinolytics in the post-partum period. Women with more severe bleeding disorders, who required factor at the time of birth were more likely to be prescribed antifibrinolytics with $68 \%$ of these women prescribed tranexamic acid compared to $24 \%$ of women who did not receive factor at the time of birth. There were no documented cases of venous thromboembolism

PPH complicated $29(22 \%)$ births with $48 \%$ of these associated with a major PPH as defined by an estimated blood loss greater than $1000 \mathrm{ml}$. Most PPHs $(26,90 \%)$ were primary and occurred in a range of IBD as 
highlighted in Figure 2 2. 12 (46\%) women had achieved levels above those recommended for safe birth, 13 $(50 \%)$ women received planned replacement factor or platelet transfusion at the time of birth and $1(4 \%)$ woman with Type 2B VWD did not receive factor replacement despite inadequate factor levels. An obstetric cause was identified in $12(46 \%)$ cases of primary PPH and included retained products of conception, vaginal wall trauma, uterine atony, placental abruption and abnormal placentation or uterine anatomy. The IBD was thought be solely responsible in $2(8 \%)$ haemorrhages and the cause was not apparent or available in $35 \%$ of cases. Secondary PPH occurred in 2 women in 3 births with 1 lady with Type 2B VWD experiencing 2 secondary PPH, one was a massive PPH; an obstetric cause was found in 2 (67\%) instances (See Appendix $1)$.

\section{Infants}

The median completed gestation was 39/40 (IQR 38 - 40) and birthweight 3325g (IQR 3060-3645). Infant diagnosis was not performed or missing in 44 pregnancies with a further 3 having genetic testing outstanding. When women with Type 1 VWD and females born to women with Haemophilia were excluded this reduced to 15 pregnancies with 6 of these infants born to women with an inherited platelet disorder. Of the infants who were tested $22 \%$ were found to be affected. Testing was initially performed on cord blood. Formal venepuncture was performed to confirm the diagnosis if the cord blood result was abnormal.

Over half $(56 \%)$ of the affected infants received Vitamin $\mathrm{K}$ and their hepatitis B vaccine intramuscularly, all without documented complication.

Discussion

Major findings

$\mathrm{PPH}$, both primary and secondary, occurred more frequently in women with inherited bleeding disorders compared to the general Australian obstetric population with observed prevalence of $20 \%$ and $2 \%$ respectively. In comparison, national estimates for primary $\mathrm{PPH}$ are $4.7-8.3 \%(6,7)$ and $1 \%$ for secondary $\mathrm{PPH}(8)$. Most striking, almost half of these PPHs resulted in an estimated blood loss greater than $1000 \mathrm{ml}$, consistent with the definition of major PPH. The increased prevalence of PPH, in particular primary $\mathrm{PPH}$, in women with IBD is reflected in other published reports with previous studies capturing HA carriers, HB carriers, women with VWD and RBD estimating the prevalence of primary PPH to be $14-22 \%$ and secondary PPH to be 2-29\%(9-12). In our cohort, PPH was experienced in women who had both "normalised" their haemostatic potential throughout pregnancy and in those who received replacement at the time of birth in a relatively even distribution. This raises the question of whether thresholds recommended in current guidelines, which are based on non-pregnant individuals are truly relevant in pregnant women with IBD. This is further supported, by the now well established evidence, that fibrinogen levels increase in pregnancy with fibrinogen levels ranging between $4-6 \mathrm{~g} / \mathrm{L}(6)$ in pregnancy compared to $1.5-4 \mathrm{~g} / \mathrm{L}$ in non-pregnant healthy individuals. Furthermore levels $<2 \mathrm{~g} / \mathrm{L}$ are thought to be associated with increased risk of PPH. This increased bleeding risk may be intrinsic to the reproductive tract as neuraxial analgesia was provided at the same threshold without any documented complication.

Any increase in factor levels achieved during pregnancy tend to return to baseline approximately 1-2 weeks post partum(4) resulting in an increased risk of secondary post-partum haemorrhage in women with IBD. Hawke et al(13) have demonstrated the protective nature of prophylactic tranexamic acid in the post-partum period against secondary PPH in these women. Whilst the overall use of tranexamic acid in our study was low, the majority of women with more severe bleeding disorders were prescribed it in the post-partum period. In addition the study spanned the period of practice change, where in 2017(14) tranexamic acid became standard of post-partum care for women with IBD.

Neuraxial analgesia was administered without complication in women with a diverse range of inherited bleeding disorders who achieved factor levels above those recommended in the guidelines, either through natural increases in pregnancy or administration of factor replacement. Unfortunately $3 \%$ (4) of eligible women who requested neuraxial analgesia were denied it. It should be noted that due to the paucity of 
safety data and in consultation with National and International Haemophilia physicians, all women with Hypofibrinogenaemia were counselled against neuraxial analgesia ${ }^{4}$. Whilst the numbers in our study are small, in conjunction with other published reports, these findings add weight to the safety of neuraxial analgesia in this population.

Neonatal outcomes were excellent with no documented bleeding complications despite interventions such as vacuum extraction and scalp electrodes being utilised in a very small number of births. When low risk neonates were excluded, including those born to women with Type 1 VWD and females born to mothers with Haemophilia, diagnosis at the time of birth was available in the majority of newborns. Recommendations for oral vitamin $\mathrm{K}$ and subcutaneous immunisations were poorly adhered to and highlights the need to improve communication between maternity and neonatal caregivers.

Strengths

Our study describes the real-life management and bleeding complications of one of the largest cohorts of pregnant women with IBD and encompasses a broad range of diagnoses, managed across several tertiary health care services in Australia.

Limitations

The major limitations of this study are the retrospective nature and method of data collection, with some missing data. The study also did not capture fertility issues including miscarriage.

Interpretation (in light of other evidence)

Our findings support previously published reports that women with IBD experience higher rates of PPH compared to the general population. The higher rate and volume of PPH suggest that target "acceptable" factor levels for safe birth, as published in current guidelines, are likely inadequate. This is further supported by the recognition that fibrinogen levels at birth are greater than in the non-pregnant population. Viscoelastic haemostatic assays, which are currently being explored in monitoring of haemostasis in PPH, may play a role in assessing haemostatic potential.

Conclusion:

This retrospective analysis demonstrates that women with IBD can deliver safely, with mode of birth dictated by obstetric indications or maternal choice, and receive neuraxial analgesia without complication when adhering to best practices.

PPH occurred at significantly higher rates and is associated with larger volumes than the general obstetric population despite adhering to current guidelines. These findings raise concern over the current definition of "adequate" factor levels at the time of birth. We feel that further study of ideal coagulation factor levels in pregnancy in normal women and those with inherited bleeding disorders are recommended.

Disclosures of interest.

Nil.

Contribution to authorship.

LJC collected data, analysed the data and wrote the manuscript.

JC critically appraised the manuscript.

BC collected data and critically appraised the manuscript.

BR collected data and critically appraised the manuscript

GKG conceived the idea, collected data and critically appraised the manuscript.

Ethics 
Ethics approval was obtained through the human research ethics committee (SESLHD HREC 15/294).

References

1. Srivastava A, Brewer AK, Mauser-Bunschoten EP, Key NS, Kitchen S, Llinas A, et al. Guidelines for the management of hemophilia. Haemophilia. 2013;19(1):e1-47.

2. National Blood Authority. Australian Bleeding Disorders Registry Annual Report. Australia: National Blood Authority; 2018-2019 18th December 2019.

3. Huq FY, Kadir RA. Management of pregnancy, labour and delivery in women with inherited bleeding disorders. Haemophilia. 2011;17 Suppl 1:20-30.

4. Dunkley S, Curtin JA, Marren AJ, Heavener RP, McRae S, Curnow JL. Updated Australian consensus statement on management of inherited bleeding disorders in pregnancy. Med J Aust. 2019;210(7):326-32.

5. Curnow J. Managing and Supporting Surgery in Patients with Bleeding Disorders. Semin Thromb Hemost. 2017;43(7):653-71.

6. McLintock C, James AH. Obstetric hemorrhage. J Thromb Haemost. 2011;9(8):1441-51.

7. Ford JB, Patterson JA, Seeho SK, Roberts CL. Trends and outcomes of postpartum haemorrhage, 20032011. BMC Pregnancy Childbirth. 2015;15:334.

8. Hoveyda F, MacKenzie IZ. Secondary postpartum haemorrhage: incidence, morbidity and current management. BJOG. 2001;108(9):927-30.

9. Kadir RA, Economides DL, Braithwaite J, Goldman E, Lee CA. The obstetric experience of carriers of haemophilia. Br J Obstet Gynaecol. 1997;104(7):803-10.

10. Kadir RA, Davies J, Winikoff R, Pollard D, Peyvandi F, Garagiola I, et al. Pregnancy complications and obstetric care in women with inherited bleeding disorders. Haemophilia. 2013;19 Suppl 4:1-10.

11. Chi C, Lee CA, Shiltagh N, Khan A, Pollard D, Kadir RA. Pregnancy in carriers of haemophilia. Haemophilia. 2008;14(1):56-64.

12. Shahbazi S, Moghaddam-Banaem L, Ekhtesari F, Ala FA. Impact of inherited bleeding disorders on pregnancy and postpartum hemorrhage. Blood Coagul Fibrinolysis. 2012;23(7):603-7.

13. Hawke L, Grabell J, Sim W, Thibeault L, Muir E, Hopman W, et al. Obstetric bleeding among women with inherited bleeding disorders: a retrospective study. Haemophilia. 2016;22(6):906-11.

14. Woman's Trial Collaborators. Effect of early tranexamic acid administration on mortality, hysterectomy, and other morbidities in women with post-partum haemorrhage (WOMAN): an international, randomised, double-blind, placebo-controlled trial. Lancet. 2017;389(10084):2105-16.

\section{Hosted file}

Table 1.docx available at https://authorea.com/users/420812/articles/527084-pregnancychildbirth-and-neonatal-outcomes-in-women-with-inherited-bleeding-disorders-aretrospective-analysis

\section{Hosted file}

Figure 1.docx available at https://authorea.com/users/420812/articles/527084-pregnancychildbirth-and-neonatal-outcomes-in-women-with-inherited-bleeding-disorders-a-

retrospective-analysis

\section{Hosted file}


Table 2.docx available at https://authorea.com/users/420812/articles/527084-pregnancychildbirth-and-neonatal-outcomes-in-women-with-inherited-bleeding-disorders-aretrospective-analysis

\section{Hosted file}

Figure 2.docx available at https://authorea.com/users/420812/articles/527084-pregnancychildbirth-and-neonatal-outcomes-in-women-with-inherited-bleeding-disorders-aretrospective-analysis 